\title{
An unusual abundance of $T$ Tauri stars? NIR study of the southern high-mass star-forming region $\mathrm{RCW} 34$
}

\author{
H. M. de Villiers and D. J. Van der Walt \\ Unit for Space Physics, North West University, Potchefstroom Campus, South Africa
}

\begin{abstract}
RCW 34 is a special star-forming region with a few stars showing an infrared excess, but seems as if it has an uncommon abundance in $\mathrm{T}$ Tauri stars. This possibility was confirmed by (i) clustering of classical T Tauri (CTT) stars in the two-color diagram, (ii) clustering of premain-sequence stars in the color-magnitude diagram, as well as (iii) the significant clustering at dimensions larger than the image-frame size, indicated by the second 'bump' in the two-point correlation analysis. A possible explanation for the above features could be the existence of an underlying wide CTT cluster with a smaller cluster centered around the massive star. The $K_{\mathrm{s}}$ band luminosity function $(\alpha=0.31)$ of RCW 34 shows that it is indeed a region of low stellar masses with an age of about of $1 \mathrm{Myr}$. Spectroscopic confirmation of such an underlying $\mathrm{T}$ Tauri cluster is necessary before any definite conclusions can be reached.
\end{abstract}

Keywords. methods: data analysis, techniques: image processing, techniques: photometric, stars: formation, stars: fundamental parameters, stars: luminosity function, mass function, stars: pre-main-sequence, infrared: stars

The full poster (in pdf format) is available at http://www.astro.iag.usp.br/〜iaus266/Posters/pdeVilliers.pdf. 